\title{
Information Systems Modernization
}

\author{
Anthony I. Wasserman \\ Interactive Development Envirouments, Inc. (DDE) \\ San Francisco, CA 94105 USA
}

\begin{abstract}
There is a huge investment in existing software systems, with organizations spending huge sums of money on the maintenance of old systems. As organizations transform the way that they do business, they must also transform their "legacy systems" to take advantage of these fundamental changes in technology. Lowering costs by moving applications to modern platforms and development approaches is a critical need for most organizations.
\end{abstract}

The need for modernization is driven by fundamental changes in the computing industry, including the movement from proprietary systems to open systems, the movement of applications from mainframes to client-server environments, and the resulting change from alphanumeric to graphical user interfaces. The transition to open client-server computing environments is occurring very rapidly, and has led to widespread adoption of standards for cross-platform networking and database access.

Modernization of systems is a complex process, with a bewildering set of possible options. Information systems managers often have essential applications running on their mainframes, and must therefore decide how to support their existing systems. There are many technical and non-technical ideas that address software modernization. These include, but are not limited to:

1) reverse engineering of source code, file, and database structures;

2) source to source translations (e.g., $\mathrm{COBOL}$ to $\mathrm{C}++$ );

3) rehosting the application by recompiling the source code on another machine, and;

4) redevelopment of the application, taking advantage of new hardware and software technology to create object-oriented client-server systems.

Modernization of application development often involves use of a different process for system development, as well as a new set of tools. This presentation provides an overview of issues in information systems modernization, beginning with the underlying technology trends and then describing various approaches to modernization. 